\title{
Mode matching and insertion loss in ultrabroadband Cr-doped multimode fibers
}

\author{
Szu-Ming Yeh, ${ }^{1}$ David Jui-Yang Feng, ${ }^{1}$ Yen-Chieh Huang, ${ }^{2}$ Tsong-Sheng Lay, ${ }^{1}$ Sheng-Lung Huang, ${ }^{1,3}$ \\ Pochi Yeh, ${ }^{4}$ and Wood-Hi Cheng ${ }^{1, *}$ \\ ${ }^{1}$ Institute of Electro-Optical Engineering, National Sun Yat-Sen University, Kaohsiung 80424, Taiwan \\ ${ }^{2}$ Department of Electrical Engineering, National Sun Yat-Sen University, Kaohsiung 80424, Taiwan \\ ${ }^{3}$ Graduate Institute of Photonics and Optoelectronics, National Taiwan University, Taipei 106, Taiwan \\ ${ }^{4}$ Department of Electrical and Computer Engineering, University of California at Santa Barbara, Santa Barbara, \\ California 93106, USA \\ *Corresponding author: whcheng@mail.nsysu.edu.tw
}

Received November 12, 2007; revised March 4, 2008; accepted March 6, 2008; posted March 11, 2008 (Doc. ID 89621); published April 8, 2008

\begin{abstract}
We investigate the fundamental insertion loss due to mode mismatch in an optical link involving a singlemode fiber-optic (SMF) transmission with the insertion of a segment of a multimode $\mathrm{Cr}^{4+}$-doped fiber (MMCDF). With an MMCDF core diameter of $\sim 15.5 \mu \mathrm{m}$ that matches the SMF, we obtained coupling efficiencies of $60.3 \%$ to $87.5 \%$ in the entire transmission spectral range of 1300 to $1600 \mathrm{~nm}$. The high coupling efficiency (or low insertion loss) makes it possible for the employment of the MMCDF as an inline ultrabroadband optical amplifier in an optical link for the entire transmission spectral range. (C) 2008 Optical Society of America

OCIS codes: $060.2320,060.2330$
\end{abstract}

Single-mode silica fibers are ideal media for low-loss optical network transmission in the spectral range of 1300 to $1600 \mathrm{~nm}$. However, the useful spectral band in a wavelength-division-multiplexing optical network depends on the gain bandwidth of fiber optical amplifiers. Current optical amplifiers, including Erdoped, Tm-doped, and Pr-doped fibers, can provide only a total of $140 \mathrm{~nm}[1,2]$. Recently, a $\mathrm{Cr}^{4+}$-doped fiber (CDF) has been fabricated by the use of a codrawing laser-heated pedestal growth (LHPG) method [3-5] or a drawing-tower technique [6,7]. The CDF amplifier may be employed in the optical network for the spectral range from 1300 to $1600 \mathrm{~nm}$. A gross gain of $10 \mathrm{~dB}$ has been achieved at a wavelength of $1520 \mathrm{~nm}$ with a pump power of $0.83 \mathrm{~W}$ [3]. However, a single-mode CDF is not available [6,7]. A multimode $\mathrm{Cr}^{4+}$-doped fiber (MMCDF) optical amplifier suffers a severe insertion loss as an inline element in the single-mode optical network. The huge insertion loss is a result of the mode mismatch and the simultaneous excitation of several modes in the MMCDF $[8,9]$. By selecting a proper core diameter, it is possible to obtain the mode matching and minimize the insertion loss.

In Fig. 1, we consider the insertion of a segment of an MMCDF in a single-mode fiber-optic (SMF) optical link. For circular fibers with a common axis, the fundamental $\mathrm{LP}_{01}$ mode of the SMF is coupled only to the $\mathrm{LP}_{0 m}$ modes of the MMCDF. If we neglect reflection at the junctions, the overall power coupling efficiency can be obtained by the continuity condition and the orthogonal property of the modes. This leads to

$$
\eta=\left|\sum_{m=1}^{M} \eta_{m} \exp \left(-i \beta_{m} L\right)\right|^{2}
$$

where $m$ is an abbreviated index representing the modes, $\eta_{m}$ is the mode field coupling efficiency be- tween the $\mathrm{LP}_{01}(\mathrm{SMF})$ and $\mathrm{LP}_{0 m}(\mathrm{MMCDF})$ modes, $\beta_{m}$ is the corresponding propagation constant, $L$ is the length of the MMCDF, and $M$ is the total number of confined modes in the MMCDF; for a typical MMCDF, $M$ can be as big as 1000 [10]. The insertion loss (IL) is thus given by

$$
\mathrm{IL}=1-\eta=1-\left|\sum_{m=1}^{M} \eta_{m} \exp \left(-i \beta_{m} L\right)\right|^{2} .
$$

If several modes are excited, the interference can occur owing to the different phases in the exponential phase factor, $\exp \left(-i \beta_{m} L\right)$, which depends on the MMCDF length and the optical wavelength. If many modes are excited, the overall power coupling efficiency can be very low and fluctuating, leading to a huge insertion loss.

A simple and effective way to minimize the insertion loss is to match the mode wave function of the $\mathrm{LP}_{01}$ mode of the MMCDF to that of the SMF. The mode matching leads to the excitation of nearly only one mode (e.g., the fundamental mode). Since both the SMF and the MMCDF are step-index fibers, it is possible to find a core diameter of the MMCDF so that the $\mathrm{LP}_{01}$ mode of the MMCDF is nearly matched to that of the SMF.

In our simulation, we employ a $3 \mathrm{D}$ simulation tool that is based on the mode propagation [11]. We as-

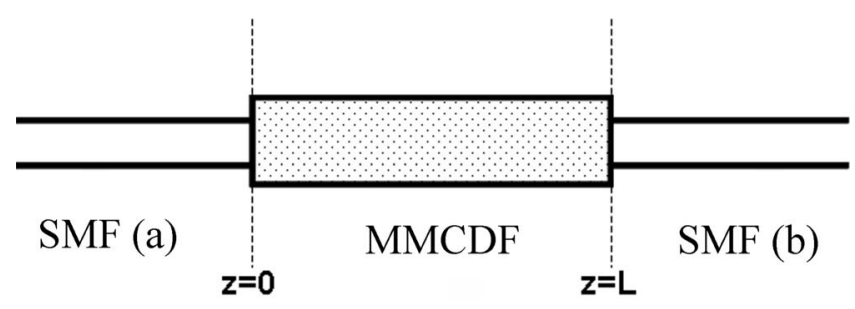

Fig. 1. Schematic of a coupling scheme of SMF-MMCDF-SMF. 
sume an SMF with a core diameter of $8.0 \mu \mathrm{m}$ and a fractional refractive-index change of $0.31 \%$. The refractive index of the core and inner clad of the MMCDFs are 1.82 and 1.64, respectively. Using the core diameter as a parameter, we carry out a numerical analysis and obtain the wave functions of the $\mathrm{LP}_{0 m}$ mode (MMCDF). We then investigate the mode field coupling efficiency $\eta_{m}$ between the $\mathrm{LP}_{01}$ mode of the SMF and the $\mathrm{LP}_{0 m}$ mode of the MMCDF. The mode field coupling efficiency depends on both the wavelength and the core diameter. Figure 2(a) shows the fundamental mode field coupling efficiency $\eta_{1}$ $\left(\eta_{m}, m=1\right)$ as a function of the core diameter for three principal wavelengths. To cover the entire spectral range of 1300 to $1600 \mathrm{~nm}$, we select $\lambda_{0}=1450 \mathrm{~nm}$ as the central wavelength. For this central wavelength, the fundamental mode field coupling efficiency is maximum at a core diameter of $14.5 \mu \mathrm{m}$ with $\eta_{1 \max }$ $=94.5 \%$. The mode field coupling efficiency drops to $59.3 \%$ for a core diameter of $7.0 \mu \mathrm{m}$. Figure 2(b) shows the high-order mode field coupling efficiency $\eta_{2}, \eta_{3}$, and $\eta_{4}\left(\eta_{m}, m=2,3\right.$, and 4$)$ as a function of the core diameter for the central wavelength $\lambda_{0}$ of $1450 \mathrm{~nm}$. Figure 3 shows the field distribution within the MMCDF at various core diameters. We notice that the field profiles are strongly dependent on the mode matching between the MMCDF and SMF. With a nearly matched core of $14.5 \mu \mathrm{m}$, the mode field size variation was significantly reduced. This is the case of a nearly single-mode excitation.

Figure 4(a) shows the overall power coupling efficiency $\eta$ as a function of the MMCDF length. The coupling efficiency fluctuates and varies from $9.7 \%$ to $70.9 \%, 83.4 \%$ to $95.6 \%$, and $13.6 \%$ to $97.5 \%$ for core diameters of $7.0,14.5$, and $28.0 \mu \mathrm{m}$, respectively. For a core diameter of $14.5 \mu \mathrm{m}$ of the MMCDF, an optimized mode matching of the $\mathrm{LP}_{01}$ mode between the MMCDF and SMF is obtained. With the excitation of a nearly single mode in the MMCDF, the interference is virtually eliminated. Thus, the fluctuation in the coupling efficiency with the length and optical wavelength virtually disappears. This leads to a low insertion loss in the entire spectral range from 1300 to $1600 \mathrm{~nm}$.

We have also carried out experimental investigations for the measurements of the overall power cou-

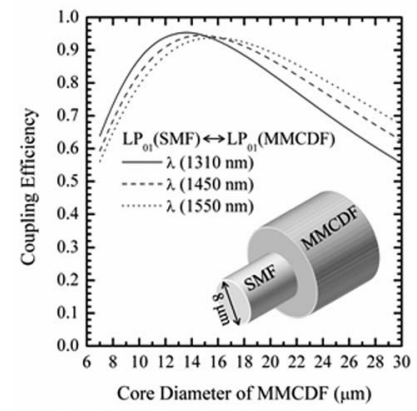

(a)

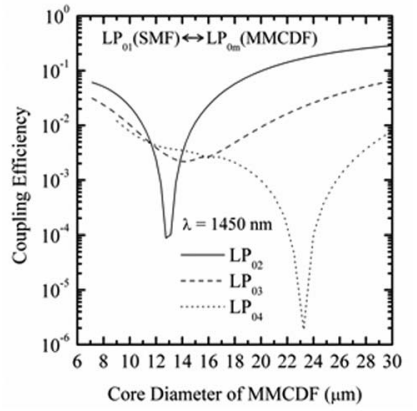

(b)
Fig. 2. Mode field coupling efficiencies between the fundamental $\mathrm{LP}_{01}$ mode of SMF and (a) fundamental $\mathrm{LP}_{01}$ mode of MMCDF; (b) high-order $\mathrm{LP}_{0 m}$ modes of MMCDF as functions of the core diameter.

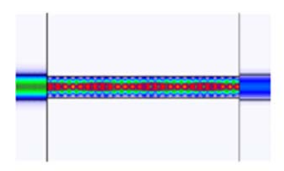

(a) diameter $7.0 \mu \mathrm{m}$

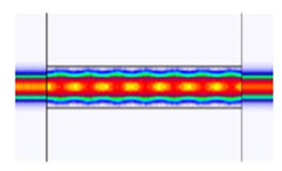

(b) diameter $14.5 \mu \mathrm{m}$

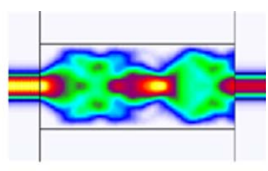

(c) diameter $28.0 \mu \mathrm{m}$
Fig. 3. (Color online) Field distribution within MMCDFs of (a) 7.0, (b) 14.5 , and (c) $28.0 \mu \mathrm{m}$ core diameters.

pling efficiency and the far-field patterns (FFPs). The length of the MMCDF segment that we used in our experiment was $\sim 1 \mathrm{~cm}$. The segment is sandwiched between SMFs as an inline transmission network. The core diameter of the MMCDF sample (I) is $15.5 \mu \mathrm{m}$ with a variation of approximately $\pm 3.4 \mu \mathrm{m}$. The core diameter of the MMCDF sample (II) is $23.0 \mu \mathrm{m}$ with a variation of approximately $\pm 2.0 \mu \mathrm{m}$. A matching fluid with a refractive index of $n=1.515$ was employed at each interface of the MMCDF and SMF to remove the reflection loss. Figure 4(b) shows the measured overall power coupling efficiency as a function of the wavelength. The coupling efficiency varies from $60.3 \%$ to $87.5 \%$ in the range from 1300 to $1600 \mathrm{~nm}$. Figure 4(b) also shows the simulation results of the coupling efficiency for the core diameters of 12 and $19 \mu \mathrm{m}$. These two core diameters are the maximum and minimum of the actual MMCDF sample (I) that has nonuniformity in the core diameter. The measured coupling efficiencies are in good agreement with the simulation results.

Figure 5 shows the FFPs of the SMF and MMCDFs that were measured at the same distance between the fibers and the CCD camera. The FFPs express the mode-matching performance between the SMF and MMCDFs. The FFPs of the matched MMCDF sample (I) with a core diameter of $\sim 15.5 \mu \mathrm{m}$ are insensitive to the wavelength owing to the excitation of nearly only one mode. The FFPs of the mismatched MMCDF sample (II) with a core diameter of $\sim 23.0 \mu \mathrm{m}$ are sensitive to the wavelength owing to the multimode interference.

As discussed earlier, the coupling efficiency is fundamentally limited by the mode matching between the SMF and MMCDF. For high efficiency coupling (or low insertion loss), the core diameter of the MMCDF can be chosen so that the fundamental mode of the MMCDF is matched with that of the

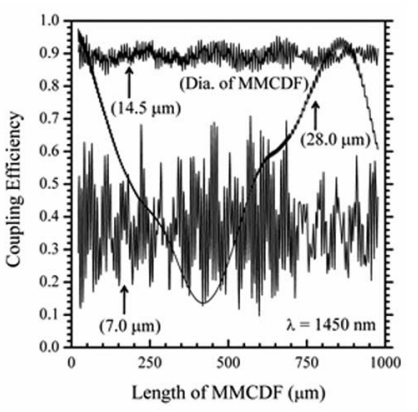

(a)

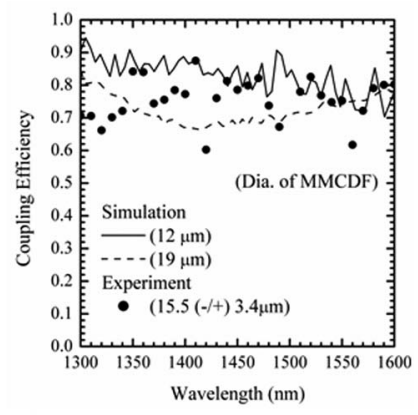

(b)
Fig. 4. Overall power coupling efficiency as functions of (a) the length of MMCDF and (b) the wavelength. 


\begin{tabular}{|c|c|c|c|c|c|}
\hline${ }_{\text {Fiber }}^{\lambda}$ & $1510 \mathrm{~nm}$ & $1520 \mathrm{~nm}$ & $1530 \mathrm{~nm}$ & $1540 \mathrm{~nm}$ & $1550 \mathrm{~nm}$ \\
\hline SMF & (C) & (C) & (a) & (2) & (C) \\
\hline $\begin{array}{c}\text { MMCDF (I) } \\
(15.5 \mu \mathrm{m})\end{array}$ & (C) & (B) & (3) & (20) & \\
\hline $\begin{array}{c}\text { MMCDF (II) } \\
(23.0 \mu \mathrm{m})\end{array}$ & & & & (C) & \\
\hline
\end{tabular}

Fig. 5. (Color online) Far-field patterns of the SMF and MMCDFs.

SMF. The mode matching leads to the excitation of nearly one mode in the MMCDF; the mode interference and the resulting fluctuation disappear. This leads to a low insertion loss and the possibility of inline ultrabroadband optical amplifiers with net gains.

In conclusion, low insertion loss is possible in the inline broadband optical amplifier (SMF-MMCDFSMF) via the use of mode matching. Our simulation results indicate that an optimum core diameter of $\sim 14.5 \mu \mathrm{m}$ is needed for the MMCDF in the spectral range from 1300 to $1600 \mathrm{~nm}$. Using a sample of MMCDF with a length of $1 \mathrm{~cm}$, we experimentally measured an overall power coupling efficiency of $60.3 \%-87.5 \%$ with a core diameter of $\sim 15.5 \mu \mathrm{m}$. The experimental measurements are in good agreement with the simulation results. This study of the higher coupling performance between broadband MMCDFs and SMFs may open up the possibility for utilizing the MMCDFs as ultrabroadband fiber optical amplifiers to cover the bandwidths in the entire 1300 to $1600 \mathrm{~nm}$ range of low-loss and low-dispersion windows of silica fibers.
This Letter was partially supported by the Department of Industrial Technology of the Ministry of Economic Affairs under contract 95-EC-17-A-07-S1-025, the National Science Council under contract NSC952221-E-110-004, and the Ministry of Education Program of the Aim for the Top University Plan.

\section{References}

1. T. Kasamatsu, Y. Yano, and H. Sekita, Opt. Lett. 24, 1684 (1999).

2. Y. Ohishi, T. Kanamori, T. Kitagawa, S. Takahashi, E. Snitzer, and G. H. Sigel, Opt. Lett. 16, 1747 (1991).

3. C. Y. Lo, K. Y. Huang, J. C. Chen, C. Y. Chuang, C. C. Lai, S. L. Huang, Y. S. Lin, and P. S. Yeh, Opt. Lett. 30, 129 (2005).

4. C. Y. Lo, K. Y. Huang, J. C. Chen, S. Y. Tu, and S. L. Huang, Opt. Lett. 29, 439 (2004).

5. J. C. Chen, C. Y. Lo, K. Y. Huang, F. J. Kao, S. Y. Tu, and S. L. Huang, J. Cryst. Growth 274, 522 (2005).

6. Y. C. Huang, Y. K. Lu, J. C. Chen, Y. C. Hsu, Y. M. Huang, H. M. Yang, M. T. Sheen, S. L. Huang, T. Y. Chang, and W. H. Cheng, in Proceedings of the Optical Fiber Communication Conference (Optical Society of America, 2006), paper OWI21.

7. Y. C. Huang, Y. K. Lu, J. C. Chen, Y. C. Hsu, Y. M. Huang, S. L. Huang, and W. H. Cheng, Opt. Express 14, 8492 (2006).

8. W. S. Mohammed, A. Mehta, and E. G. Johnson, J. Lightwave Technol. 22, 469 (2004).

9. D. H. Sim, Y. Takushima, and Y. C. Chung, in Proceedings of the Optical Fiber Communication Conference (Optical Society of America, 2007), paper OTuL3.

10. A. Yariv and P. Yeh, Photonics, 6th ed. (Oxford U. Press, 2006).

11. FIMMPROP, version 4.3, Photon Design, Oxford, U.K. (2004). 